\title{
Self[ie] Reflective Practice: revealing student engagement through the photographic performance of the self
}

Jackson, H. (2019). Self[ie] Reflective Practice: revealing student engagement through the photographic performance of the self. Learning, Media and Technology, 44(2), 144-161.

https://doi.org/10.1080/17439884.2018.1563107

Link to publication record in Ulster University Research Portal

\section{Published in:}

Learning, Media and Technology

Publication Status:

Published (in print/issue): 03/04/2019

DOI:

10.1080/17439884.2018.1563107

\section{Document Version}

Author Accepted version

\section{General rights}

Copyright for the publications made accessible via Ulster University's Research Portal is retained by the author(s) and / or other copyright owners and it is a condition of accessing these publications that users recognise and abide by the legal requirements associated with these rights.

\section{Take down policy}

The Research Portal is Ulster University's institutional repository that provides access to Ulster's research outputs. Every effort has been made to ensure that content in the Research Portal does not infringe any person's rights, or applicable UK laws. If you discover content in the Research Portal that you believe breaches copyright or violates any law, please contact pure-support@ulster.ac.uk. 


\section{Self[ie] Reflective Practice: revealing student engagement through the photographic performance of the self}

This paper positions the photographic image of the self (the 'selfie') as the reflexive indicator on which students are prompted to reveal their learning behaviours, and uses this mode of reflexivity to construct a qualitative study of engagement in a higher education setting. The study leverages the intensified processes of self -reflection created by the novel forms of convergence across the social landscape in which the selfie is embedded, to reveal how oppositional and indexical selfie-reflective practices can operate to uniquely connect behaviours to learning in this sector.

\section{Introduction and context}

Gathering and evaluating data concerning the performance of the student, has become part of the contemporary pedagogic landscape in higher education in which course teams and tutors seek to extract information to monitor and improve the quality of teaching provision. Capturing authentic information pertaining to student learning behaviours in order to create key indicators that map these behaviours to high quality learning outcomes, requires students to reflect on their past activities and behaviours in the learning environment and feed forward the result of this reflective operation.

Coates (2005) warns that there are a number of challenges that impede the validity of data extracted from the reflections of the learner. Many students 'sleepwalk' through their practice of learning; the classroom experience for the student becomes a purely mechanical function without cognizant awareness (McIntosh 2010). Students have been found to create false recollections in an effort to communicate an experience that is more desirable and/or is expected from them within the particular institutional context of self-reporting (Paulhus 1984), and as Porter's study highlights (2011) comprehension of the reporting question may not be universal across a body of students. 
Effective and meaningful reflective practice in any sphere requires the participant to engage in a culture of observation and critical dialogue about the observed and objectified self (Van Manen 1995). The process of reflection is understood as both a skill (Schon 1987) and a process through which the student is able to gain knowledge about their performance to enable them to progress their learning (Jones 1995). Studies of reflective practice across a number of educational contexts reveal that students more generally in the self reflexive process, lack the skills required to carry out this process effectively. In their assessment of the literature on reflective practice within the nursing profession, Atkins and Murphy (1993) identify that the principle skills needed to become reflective are: self-awareness; critical analysis; synthesis; and evaluation. Without these skills, those that participate in the process are: unable to identify what works well for them; convert experiences into appropriate verbal propositions; or produce adequate accounts of their past behaviours (Jones 1995; Van Manen 1995; Harford and MacRuairc 2008; Schon 1987).

Philosophical arguments are used to underpin the process of reflection as a construct. Dewey's $(1933,14)$ central argument in How We Think, in which he describes the process by which we rationalise the complexity of what it is we know and understand, highlights that when asked to create verbal propositions about our experiences, this creates within us "perplexity, confusion and doubt." Ambiguities and dilemmas are created by such a confrontation. Dewey posits that where this uncertainty and doubt exits when asked to recall facts and memories, we commit to an act of looking to identify physical materials that will help prompt a memory or resolve a doubt. This,

"Looking brings into facts before the mind that enable a person to reach a conclusion on the basis of evidence...the act of looking [is] deliberate, [is] performed with the intention of getting an external bias on which to rest a belief" (13). 
Pedagogic practice has often used visual inquiry to inform the knowledge process, using images as stimuli to prompt recall and memories that connect to an informed qualitative experience of the self (McIntosh 2010; Grushka 2010). Exploring the visual as a sociological knowledge and a critical text to prompt our sense of knowing and being, this study thus leverages the potential of the image to intervene in the reflexive process of knowing the student self. Deploying a visual ethnographic methodology, the study positions the 'selfie' as the reflexive visual stimulus on which relevant information and authentic insights connected to student learning can be explored.

The selfie as a cultural artefact, is part of the social media landscape that exists in contemporary culture in which the photograph of the self taken by the self and posted online, forms part of the habitual behavior related to the process of socializing through communication networks. Today, students articulate their identity as social beings by uploading photographs to document their lives, and through social media networks (for example Facebook, Twitter, Instagram) they become part of a social community through photographic exchange (Van Dijck 2013).

The transformative potential of using selfies to stimulate reflective practice is underpinned by the way in which the photograph of the self, when enfolded into the dynamic interfaces of social networks, is able to make the connections between personal memory and the routines of everyday life, visible and explicit (Hand 2012). The photographic image has always been an important artefact upon which acts of remembering are performed (Spence and Holland 1991), and has contributed to notions of personhood, self knowledge and the truth (Pink 2001; Rose 2016). However, while the act of looking at oneself in an image has always been a rich act of self reflection and contemplation, the selfie reveals new contexts in which the visual and textual object of the self is performed. 
When the image of the self is subject to forms of enhancement and description associated with the social media distribution tools and processes, this requires the author of the image to enter into a negotiation with the image in order to perform acts of classification on the image of the self for the purposes distribution and communication. There is an agentic force at play; applying modes of classification pushes the image author to act and reflect in order to create definitions for oneself. As Foucault highlights in Technologies of the Self, framing oneself as a subject in order to create a definition of oneself for others, requires an intensity of effort. To define oneself, an examination of the self and conscience, and the act of remembering the self, are required to be performed and assimilated.

The cultural coding mediated by the communicative practice of the selfie, in which the self-conscious act of enhancement and description associated with the posting the photograph of the self, facilitates a practice of looking at the self in which the self is both the observer and observed. Where self-doubt and dilemmas of what it is we know and understand the self to be may open up a gap in memory, this is resolved through an embodied practice of the self as a subject of observation and description. The complex process of practices that construct what is known about the self, and the intensity of effort that this involves, is underpinned through a visual system of simulation of the self as a subject. The photograph of the self moves from being isolated evidence of the the indexical self, to an image that functions within the interpretive field. As Robins (1996) highlights, through the simulation of the self as a subject, it becomes possible to experience the self as an object and "interact with it as if it were an object in the real world... and when this becomes the case, we can say that we 'know' the object in more complex and comprehensive ways" (156). Therefore, leveraging this intensified process of knowing oneself as a subject through the practice of looking at the self image, connecting the selfie to self-perception and self-identity, has the 
potential to create a mediated process of self-reflection that is more self aware and/or self conscious, and therefore more successful as a practice of knowing the self.

\section{Research Questions}

This study positions the selfie as the visual stimulus upon which students are asked to perform an effective reflective practice of knowing oneself as a learner. The aim of the study is to identify how the messages that are revealed by the selfie-reflective act, operate to reveal critical and synthesized information relating to the performance of individual students. Through assessing the form and practice of the selfie to understand how it can communicate effective messages that relate to the learner, the study positions three research questions:

1. How does the selfie (a photograph of the self, taken by the self using a mobile phone camera, then annotated and posted to Instagram) operate as a reflective stimulus within the sample of 33 students studied?

2. How does the reflective practice performed with and upon the selfie construct messages that relate to a grammar of student engagement (e.g. satisfaction, achievement, involvement with learning, and perception of control)?

3. What do these messages tell us more generally, about learner engagement?

\section{Literature Review}

The corpus of research dedicated to the selfie seeks more generally to position the selfie as a form of strategic self-representation that operates to disseminate a desired impression to others (Schleser 2014; Dobson 2015; OkLyu 2016); document a specific moment in one's life (Chen et al. 2016; Sung et al. 2016; Murray 2015); and establish a connection, or act as a witness to both incidental and significant events (Maniou and Veglis 2015; Ibrahim 2015; Van Dijck 2013; Koliska and Roberts 2015). 
Quantitative studies have identified clear trends in the practice of selfie-taking across the generations and genders. Young people are more likely to post selfies than adults (Jang et al. 2015), and the behaviors revealed in the selfie image analyzed in conjunction with usage data and contextual metadata, focus on a form of self-expression that operates to communicate an emotional state (Zappavigna 2016). Across genders, women are more likely to post more selfies than their male counterparts (Sorokowska et al. 2016), with the practice of selfie-taking presumed to be more acceptable as a female activity (Burns 2015; Williams \& Marquez 2015).

Pounders et al. (2016) highlight the desire to be 'liked' as the key motivator that drives selfie-postings among female millennials, with self esteem playing an integral role in selfie posting behavior. Based on the number of 'likes' received from a post, self esteem is either enhanced or diminished; self esteem is thus operating as both the motivator and the outcome of the activity. Cyberpsychologies highlight the intensity of such motivations, positing that girls are addicted to likes (Mascheroni et al. 2015). The performance of the selfie amongst females, is widely understood as a self-objectified form of behavior in which the female "internalizes an observer's view of their own bodies as an object to be evaluated," (Cohen et al. 2018, 69).

This objectification and search for esteem through the selfie have fed into claims that young women are responsible for a culture of narcissism operating in contemporary society (Dobson 2015; Williams 2016). However, there are a growing number of empirical studies operating to counter such gender positions. Sorokowska et al. (2016) has provided evidence that the link between narcissism and selfie-posting behavior is comparatively weak among women than men. Examining selfie-posting behavior from a personality perspective, Sorokowski et al.'s (2015) empirical study highlights that extroversion and social exhibitionism drive self-presenting behaviors in both men and women. 
As a tool that can enable impressions to be managed and when deployed as a form of strategic representation, Murray (2015) argues the selfie enables counter arguments to be made against hegemonic norms associated with gender. Those frameworks that deploy a Post Feminist ideology to the analysis of the characteristics associated with selfies posted by women, highlight the intentional control of the selfie taker, positioning a practice of the selfie in which women seek to gain control over their body image (Burns 2015; Dobson 2014; Jerslev and Mortensen 2016; Barnard 2016).

The affordances of the selfie operating to generate discourses related to empowerment reveal more non-generalized approaches to understanding the role of the selfie in the formation of self identities online. William's and Marquez's (2015) study of male Black and Latino selfies reveal a representational image that operates to deflect racial prejudices from non-minority groups through the creation of oppositional identities. Toffoletti and Thorpe's (2018) analysis of female athletes' self-representation highlight how the visual message constructed by the athletes work to counter normative gender and sexual identities commonly found within sport, including both sexual and racial aspects. Wargo's (2017) analysis of how young people explore their gender and sexuality, positions the selfie as a means of giving a voice to difference. However, there are also paradoxes encountered within these claims of empowerment. As Bernard (2016) surmises, the control exerted by the individual in the conscious construction of the desired presentation of the self, whilst operating as a means to empower the individual, can often simultaneously conform to the hegemonic norms that it is seeking to revise.

As a highly presentational form of self representation, semiotic modes of analysis are most often applied to the selfie. The overwhelming corpus of research identifies with a visual practice of self-representation that is deliberate and calculated; the impression projected through the selfie is carefully managed by the intentional agency of the author of the image. 
There are a small number of studies that have attempted to position the selfie as a sociotechnical phenomenon, to understand a more nuanced relationship between the user and the technology, arguing that the allure of the selfie rests not on the visual, but on the wider sociotechnological practice of the image of the self (Cruz and Thornham 2015). As Frosh's (2015) gestural image positions, the selfie implicitly implies the role of the self in constructing the image, "drawing attention to the immediate context of image viewing and to the activity of a viewer" (1615). Selfies thus are understood to co-exist with human creativity and agency (Ibrahim 2015), promoting a self image operating as a connective performance rather than a visual sign. It is therefore the exchange of messages that becomes the symbolic representation.

This is uniquely captured in Jerslev and Mortensen's (2016) study of celebrity within the selfie phenomenon. Analyzing how the Danish songwriter and pop singer Medina uses selfies to connect with fans, Jerslev and Mortensen observe that to compose the selfie, the performer is required to step out of her activities and routines in order to experience herself in the moment of image capture.

"The selfie captures a posing body, lingering in a performative space and time in between, taken by the camera and yet in the very process of being taken, immobilised but not quite stilled, situated in a moment of presence." (255)

Watching Medina watching herself, "connects and communicates with fans through displays of moments-in-between, which are intimate and inviting, because we watch the image as it comes into being" (260).

The allure of the selfie is thus better understood, not as an image of self representation nor a single image upon which semiotic codes of analysis can be applied, but rather as a mode of visualization that brings the self into being. As such the selfie becomes an effective point of departure on which new meanings can be communicated (Mirzoeff 2016); a vehicle 
to expand conversations about the meaning, significance and contested nature of knowing oneself.

\section{Methodology}

To capture the ideological contexts that frame the selfie as a reflexive performance of the self, this study analyzed 105 selfies posted by 33 research participants (18 females and 15 male). To determine a performance of the self against a framework of engaged learning, these Year 2 and 3 media students were required to take a number of selfies over the course of a semester, within the learning environment in which they were being taught.

Participation with the study was wholly voluntary. As identified in the literature review, self-posting behaviours are critically linked to issues of self-esteem, hence a private Instagram account was constructed for the project so that only those participating in the project could view the posts. Students also reserved the right to remove the post once the study was completed.

There were four acts to be performed by the participants in the project:

1) Capture a selfie using their mobile phones.

2) Reflect on the selfie, examining the self image to prompt a consciousness about their learning performance at that moment.

3) Provide a description of the observed self, capturing the act of remembering in a short annotated statement.

4) Post the selfie and its description to Instagram.

In order to understand the way in which the learner engages with the selfie as a process of reflection, the analysis of the reflective practice (that includes both the performance of the self in the image and the knowledge of the self revealed in the ascribed annotations) operates as an ethnographic form of research. Ethnographic approaches as described by Algan (2008), help to present a synthesized account of the aesthetic and functionalist effects of socio- 
technological forms, and address the situatedness of the specific context in which it is used. Coleman (2010) however, in her survey of the ethnographic corpus in digital media, warns that the forms of visuality mediated by digital media pose challenges to ethnographic analysis. Problems in deciphering these visual forms arise from a variety of characteristics of its form including "anonymity, modalities of hypermobility, ephemerality, and mutability" (494). Responding to these challenges, Coleman recommends that in the examination of what on the surface may appear to be a single nuance in a type of digital mediation, the "devil is in the detail" (497), and if approached through this acknowledgement, the analytical framework has the potential to provide a dynamic view of knowledge and subsequently deliver significant cultural and social conclusions.

"Theses details are often aesthetically valued for revealing the splendor of sociocultural life and at times are also ethically deployed to push against the faulty and narrow presumptions about the universality and uniformity of human experience" (497).

To understand how the human experience under analysis operates to inform pedagogic discourse, learner engagement is positioned as a measure of the processes and methods by which a student is engaged with their learning (Trowler 2012). As a pedagogic framework for engagement, this study deploys the psychological perspective which views engagement as an internal individual process and measures how students are feeling (Kahu 2013). The analysis of learner engagement performed is framed by a pedagogic grammar that identifies a topology of engagement that moves from high level interest to high level of rejection. The reflexive performances stimulated by the selfie are therefore assessed against a matrix of engagement indicators created from a synthesis of the pedagogic literature (Bryson 2014; Coates 2005; Byrne and Flood 2005; Kahu 2013; Wehmeyer et al 2000; Zepke and Leach 2010; Astin 1984). This includes as a sliding scale: the interlocking of institutional 
interests, goals and aspirations; persistence and sense of empowerment; sense of control; sense of identity attached to course; general signs of involvement with learning; general enjoyment /interest in task; withdrawal through disinterest; passive response to alienation; negative perceptions of control; active response to alienation/ conflict; strong negative emotions displayed.

\section{Mode of analysis}

The analysis of the resulting selfies posted to Instagram was mapped to an understanding of how the participant was performing a pedagogic mode of engagement, where the performance is defined as all of the intentional activity of the informant posting the selfie to Instagram. As a process this considered:

- How the image of the self was posed/ composed (the closeness of sitter to frame; the direction of gaze; the inclusion of other people and props within the frame; the depth of field within the frame; the emotion exhibited by the sitter);

- If and/or how the image was edited (the application of filters; inclusion of graphic or other photographic elements; any other authoring of the image that required image manipulation);

- The messages revealed by the annotation (words and phrases that related to their studies including coursework, academic calendar, peers, instructors, achievement, emotional state);

- The messages revealed by the additional metadata (emoji and hashtags that related to their studies, coursework, academic calendar, peers, instructors, achievement, emotional state);

- The academic standing of the author of the selfie (using performance measures including attainment results, the author of the selfie was identified as either a high or low achieving learner within the cohort of students studied). 
The results of these five modes of analysis were used to frame an understanding of how the self image was operating as a visual practice upon which the self refection was being performed (Table 1). The self refection that was being performed, was then mapped to the engagement matrix (previously identified in the methodology) to identify how the selfiereflective practice of the participant created specific pedagogic messages (Table 2).

\section{[Insert Table 2: Selfie Reflection Engagement Matrix]}

As this ethnographical methodology is inherently concerned with the interpretation of images and the reflective consciousness that is attributed to these images, it is important to consider the rhetorical contexts in which the images are imbedded; the context in which the images are being produced; and the form in which they are distributed. Thus the analysis in this study deploys a digital visual cultures theory to understand how the selfie facilitates a mode of reflexivity, rather than theoretical approaches that are solely connected to a semiotic mode of analysis. Adapting the Lister and Wells $(2000,64-65)$ framework for analysis that incorporates these paradigms, the main features of the analysis of the selfie-reflective practice includes: addressing the cycle of production, circulation and consumption of the selfie through which meaning is articulated and transformed; acknowledging the image's specific material properties and the medium of the technology through which it is realized; reviewing the actual social process of looking at an image; analysing the codes and signs deployed by the representation through which meaning is constructed and understood; understanding that the image cannot be reduced to a singular meaning; and promoting a process of looking that is fundamentally embodied.

Consequently, as the selfie-reflection constructs a reading of the self that is socialised, the outcome of the reflective practice is understood to operate from a position in which the student is cognisant of, and moulds an image of the self to fit the expectations of an audience. 
Goffman's (1959) theory of self performance highlights the limitations of the study; the presentation of the self is ultimately performed according to the expectations of the society in which it is operating. As the author of this study is known to the participants primarily as a course instructor, the research participants may be conveying a view of a learning situation that they feel is acceptable to the author. Consequently, what is read in the images and annotations constructed through posting the selfie, is approached from a position in which the participants are understood to guide and control the impression that is formed. It is cautioned in the analysis therefore, that the motivation for creating a particular impression will involve aspects of concealment and enhancement in relation to the learning behaviours revealed.

Furthermore, social media platforms (in this instance Instagram) do not act as neutral tools in the staging of the self(Van Dijck 2013; Duguay 2016). The technology that facilitates the published image online, works to promote formats that communicate and intensify emotional framing of the posted selfie image (for example enabling the author of the image to add textual descriptions, emoji and hashtags to the image posted), and create affective ties between the subject of the selfie and audiences within these networks (enabling the other research participants within the closed Instagram group to 'follow' the author of the post and post responses). These technologies thus will also be considered from a position that they may also be deployed within a particular context and motivation to conceal and/or enhance what is being communicated to the participants' peers.

\section{Findings}

\section{Selfie-reflection, an oppositional and indexical construct}

The analysis of the practice of the selfie in the first instance sought to identify how the participant was using the self-image to make a connection between themselves and their learning (Table 1). This analysis began with identifying the codes and visual signs deployed in the self image; applying a semiotic mode of analysis; and then mapping these signifiers to 
the contextual characteristics of the reflective annotated description that accompanied the self image. By identifying how the intentional agency of the author was working through a representational practice, the analysis revealed that there are two very distinct self-reflection strategies operating within the sample studied: the selfie that operates to enable the self to acquire and assimilate information about the self; and the selfie that operates to state, confirm or manipulate the reflexive messages communicated.

In the former, the individual self becomes a subject of observation, upon which a reflective act is performed (Picture 1). The image constructed by the selfie is essentially a visual presentation of the student, a mirror image. These images are devoid of the visual appearances that offer any kind of self promotion or mediated appearances. The framing of the image captures the sitter close to the picture frame, often leaning into the picture frame, displaying neutral expressions and with minimal inclusion of the wider learning environment. Without obvious agential intention, the image operates as a material artefact and durable form of self-representation upon which there is a subsequent examination of the self with respect to what is seen in the image. Self-consciousness is prompted by considering the image of the self, and the boundaries of the self defined by reflecting on the selfie are fed back to the self. The reflexive act of knowing oneself operates post image capture, and remembering the self as a learner is performed upon the selfie image. In this sense, within the act of reflection and experience of the self, there is an understanding of the self in opposition - between the external self that is seen, and the internal self that is felt.

\section{[Insert Picture 1: Oppositional Selfie Practice]}

[Insert Picture 2: Indexical Selfie Practice]

In the second self-reflection strategy, what is known about the self is being performed at the moment of image capture (Picture 2). Reflection as a construct has been calibrated for the camera. These images identify with the social-structural and symbolic nature of photographies in which the selfie operates as a social practice, a message to be communicated 
to audiences. Within these images there is an intensive self-conscious distribution of the identities that are being performed. These selfies deploy props (including textbooks, laptops and spectacles) captured within the frame of the sitter to emphasise the practical aspects that connect the known self narrative to the presumed visual representation of a learner. In this sense of knowing the self in order to create an indexical self definition, the management of appearances connects the sitter to a rhetoric of learning.

The framing of emotion is key in the visual and textual elements in these selfies. The focus of the performance emphasises the emotion that is being consciously felt, but invisible within the context of the learning environment. The learner is bringing into being consciousness internal messages, and what is being felt is made visible for the purposes of documentation and communication.

These alternate selfie-reflection strategies have an impact on any attempt to form conclusions based upon a semiotic analysis of the selfies, and reveal more nuanced variables of reflection as a construct when mapping the semiotic study across the matrix of engagement. Where the selfie operates a reflexive object enabling the self to acquire and assimilate information about the self, the sitter more consistently performs a neutral expression. There is an intensity in the expression, a sense of looking into, and communicating to oneself. In contrast the selfie operating to communicate an indexical identity, reveals sitters displaying a wide range of emotions. While some of these emotions appear spontaneous (wide smiles communicating contentment), others are clearly constructed and controlled (wide-opened eyes communicating anxiety and horror). These emotional variances cannot therefore be understood to relate to exclusively to authentic emotional conditions of the sitter at the moment of image capture, but need also to be considered in relation to how the learner is using the selfie within the process of reflection as a construct. 
Understanding how the selfie operates either as a form of seeing oneself or knowing oneself, is also significant when projecting gender variances revealed through visual signs. Visual analysis of the selfies reveal that the female participants communicated a broader range of visible emotions, across what would be understood to be positive, neutral and negative emotions. In contrast, the male participants exhibited a much narrower range of visible emotions, with almost half of all of the male informants consistently performing neutral emotions. However, the male participants in this study were more likely to use the selfie as a reflexive text upon which knowledge of the self is constructed, and females in the study more likely to perform an indexical self that is already known. Emotion for the male participants, is experienced by reflecting on the selfie, for the female participant emotion is congruous with the selfie construction.

\section{Grammar of student engagement, an intentional, situated and temporal construct}

There are a significant number of practices that can be identified across the sample studied, that connect the performance of the learner within the reflective act, to a grammar of learner engagement. The practices highlight how compositional techniques revealed in the selfies operate to communicate specific learning relationships, and demonstrate the impact of the immediacy of the situational context and temporal specificity of the reflexive act performed. While these aspects are revealed through textual and visual elements, the analysis highlights that these practices only operate as effective and distinctive features when they are understood as indicators that are entwined and bound with a complex process of practices that performatively construct the selfie reflection.

The first of these practices to be considered in relation to how the selfie-reflection communicates certain messages relating to learner engagement, is how the composition of the selfie image operates to frame either an individual sense of the self as a learner, or one that is connected to others and/or the institution. 
The compositional element of the selfies in this study are predominantly characterised by close cropped portraits of the sitter close to the picture plan. Within this range of closeness there are distinct variables. Female participants were more likely to fill the picture plan with their self image, engaging in compositions where the portrait exceeds the dimensions of the screen and is cropped by the frame of the camera (Picture 4). The resulting composition thus obscures any background detail. In contrast male participants were more likely to lengthen the space that is represented by composing the portrait so as to include the visual details of immediate surroundings (Picture 5). Contained within the backgrounds to these self portraits is the furniture and physical debris that constitutes their learning environment.

There are conflicting discourses connected to the language communicated by the space inhabited by the engendered subject of the image. Gender politics abstracted from visual media discourse highlights that the shortening of the picture plan is most usually attributed to female representation, and is communicative of a more submissive and lower status subject (Henley 1977). This discourse however has arisen from studies of within visual culture (largely film and advertising) where the positioning of the female subject is subject to directorial control. Visual discourse arising from the selfie phenomenon posits that the more physical space someone takes up with their body when constructing a selfie, the more confident they feel and/or will appear (Cuddy 2012).

The close portraits of the females in this study (used in both oppositional and indexical selfie-reflections) communicate a range of emotions, both positive (for example happiness and confidence) and negative (for example worry, fear and anxiety). Therefore, when filling the frame with a close portrait, it is the performance of the self that may be presumed to be confident (as opposed to communicating confidence as an emotion). Yet as the study also reveals, where the male participant uses a framing composition that lengthens the picture plane, these also communicate confident performances (Picture 5). 
This visual analysis of the selfie only becomes meaningful once it mapped to the situatedness of the context of the performance; in this instance how the composition of the portrait that lengthens the picture plane to reveal the learning environment operates to communicate the sitter's connectedness to their learning. Including laptops, desks, the lab computers, other students, the architecture of the learning space, the instructor etc., performs messages that connect the experience of being a student to the learning environment and/or the wider student cohort. This analysis is confirmed when the visual aspects of the performance of the self is read in conjunction with the written descriptions posted with the image and mapped to a grammar of engagement. For example, the annotated description accompanying the male learner in Picture 5, “Guess who's back? back again...” reveals a narrative of a student returning to university after the Christmas vacation. The emphasises and distinction created by being "back" in a learning space after a period of absence frames a context learning that is appraised by a sense of belonging to the institution.

At the opposing end of this dichotomy, the female learner in Picture 4, who creates a proximity of closeness of the self to the picture plane so as to exclude the situated learning environment, performs a self-reflection that centred wholly on the self. The annotated description and hashtag attributed to Picture 4, confirms a performance that is wholly internalised, "\#feelingmotivated". While there is consistency in this assessment across the study, it is cautioned that this visual staging is not gender specific. Both male and female participants constructed self images in close proximity to the picture plane, (and these were the largest collective category in the samples collected through the study) that collectively communicated an individual sense of self within the learning experience. Slight gender variances were however noted in the way in which the female learner uses this close framing when constructing an indexical self, with the female learner was more likely to deploy 
personal props (spectacles, satchel handle visible across the shoulder, text and exercise books held close to the face) to create a self-reflection that is communicates the self as a student (Picture 2).

Within the realm of closeness to the picture plane, there are further distinctions within the compositional techniques: students who achieve the sense of closeness by holding the camera close to the face to compose the framing, and those that clearly appear to lean into the frame. Where the student leans into the frame, the visual depiction is much more selfconscious and oppositional in its presentation, and there is an intimacy created by reducing the distance between the sitter and the frame.

In those images where the learner has sought to expand on the space represented in the image, this often operates to include another individual within the framing (Picture 6). The other individuals captured within these selfies are not random encounters; the framed colearner is someone with whom the selfie-taker has collaborated with on group projects and/or presentations and therefore is someone with whom the selfie subject is understood to have an affinity with and shared values. In these images there is a shared identity, with the two informants co-operating to perform an identical indexical performance. The importance of the types of learning relationships expressed in these instances is highlighted by Zhao and Kuh (2004). Working co-operatively and collaboratively with peers has been identified as having a positive affect on a student's personal and social development, enhancing their sense of belonging and subsequently improving engagement.

[Insert Picture 6: Shared Self Performances]

A second characteristic that frames messages relating to learner engagement is the nature of the temporal and situated learning behaviours demonstrated, that works alternately across the genders. The annotations constructed as part of the reflexive process, reveal that student involvement with their studies is both highly generalized, with comments about the 
general student experience, ("Feeling like a pancake - deflated and tired, but none the less will power through"), and highly specific, with comments referring to particular coursework and classroom activities, ("Another presentation done, hate them”). While the balance between general and specific comments in the male participants was weighted slightly more in frequency towards generalized comments, overwhelmingly the female participants described an involvement with their learning that was highly specific to the immediate learning experience. Documenting activities and experiences as part of the selfie-reflection process, these females reported immediately preceding incidents to narrativize an emotional sense of self at the moment of image capture, ("We had intervention presentations in class today. I hate presentations. \#Nervous"). Understanding student involvement using Astin's developmental theory (1999) that is concerned with how students are involved with their studies, reveals that these motivations and behaviors in the female learner have a focus on the immediate and specific learning encounter; they dwell on the immediate task in hand. These learners frame their reference for describing how they feel about their learning more generally, by reacting to circumstances in the present. In contrast, the male participants in this study were more likely to reflect on an accumulation of learning experiences beyond the immediacy of the moment in which the reflection is occurring, "Work's really starting to pile up \#piling up \#work \#coursework."

While gentle reminders were given to the research participants each week to encourage them to post a selfie for the purposes of analysis, the greatest rate of participation was observed at times when specific academic milestones and tasks were required from the student, such as class presentations and coursework deadlines. Around the timings of these milestones within the academic calendar, the posting and images reveal a variety of strategies by which the learner engages with their learning. There are those students for whom the emotional intensity of the learning activity resonates most significantly, with the self 
reflection capturing very active responses to the sense of conflict that is created, ("Deadline Day got me like...", posted with an image of the participant with a highly exaggerated downturned mouth). For some students, a grammar of engagement connected to withdrawal is identified as a coping mechanism for the anxiety created by the pressure to deliver learning outcomes, ("When you realise you have a lot more work than you thought, so you go to [the local night club] instead"). Other students reveal an understanding of their anxiety created by workloads as a temporal condition, finding motivation by focusing on a point beyond their studies, ("Exhaustion is finally starting to set in, just a few more weeks until summer, \#wegotthis").

While anxieties are understood by the learner as having a temporal condition in this instance, time forms an emotional resonance across the cohort of students. In a number of annotations, the emotional performance of the student was assessed against a weekly semester temporal indicator, as the student consciously positions their engagement with learning within a temporal framework, (“Week 8, wait week 8 already?”; “Week 8: Slowly becoming more and more stressed!").

\section{Student engagement, autonomy, anxiety and taxonomies of control}

Most consistent in the assessment of the reflective descriptions tagged to the selfie, is the performance of a student body that has a very clear understanding of what is expected of them at this level of study. As a collective narrative that constructs a grammar of student engagement, the annotations reveal an effort by the student to deliver work on time, deliver work of a high standard, and succeed on their course of study. Individual expectations are evident and most profound in the reflections, and reveal the importance and significance that students place on achieving academic goals. Therefore, the measure of student engagement that is being revealed in the reflexive performance that correlates directly to an engagement indicator (a clear interlocking of institution and individual goals), is certainly very high. 
However, what is compelling in the reflective statements, is how the individual narrative of the self constructs a performance in which the responsibility for achieving these positive learning outcomes is on the learner, not the institution.

These largely autonomous experiences reported in the reflexive process of the self, also present a mode of knowing oneself in which the emotions that are expressed operate a taxonomy of control (Skinner 1996; MacCann et al. 2011; Schutz and Davis 2000). Where the student performs an anxiety in reflecting on their learning using the selfie, the student proposes actions that will change the emotional conditions documented at the point of image capture and create new conditions in which they will be able to succeed, ("Trying to get more research done for my first mini project, slightly lacking it motivation but plan is to work hard to get everything done"; "Feeling ready to start some mini projects even if I'm slightly lacking in motivation”. This type of extrinsic motivation as highlighted by Zepke and Leach (2010) brought about by the desire to achieve academic goals, becomes part of the strategy by which the student is attempting to overcome the existing negative emotions that are hindering their academic performance. The selfie annotations are operating in these instances as auto-ethnographic statements of the student's perceptions of their own efforts to actively construct knowledge and there is a clear willingness to take personal responsibility for the academic preparedness. Where the examination of the self created by the reflection upon the image of the self reveals self doubt, self-regulation is performed as an attempt to work against the emotional condition.

In contrast, where the control value is absent in the annotations that reveal negative emotions, a sense of hopelessness is inferred ("Feeling anxious and confused for this year's modules"; "Deadline Day got me like..."; “Tired or confused?”). To ascertain the impact of these control strategies and assess how or if the absence of an extrinsic motivation indicator affects the academic performance of the student reporting, it is noted in this study that when 
those female participants who would be identified as high achievers academically (using grades as a benchmark of success) performed negative anxieties, these were more likely to be subject to a taxonomy of control. Here the emotional involvement operates between the opposing states of an anxiety of performance and determination to succeed. The extrinsic motivation frames the dynamic context of the emotional range, essentially forming a strategy by which the student can move from a place of negativity to a condition that may be perceived as more positive, hopeful or confident. In contrast high achieving male learners constructing visual and/or textual messages that communicated a performance of anxiety, were less likely to frame the negative emotions within a control mechanism. The anxiety of the performance is reported, but is not subject to a determination to intervene in the negative emotional involvement reported through the selfie-reflection. For these high achieving male performers, they were more likely to communicate a performance of the self that downplays or underplays their academic abilities. The observation of an engendered use of control to regulate emotions, was also consistent where both male and female students identified within this study as less academically achieving; the taxonomy of control was more likely to be absent in the male learners performing the anxiety.

The occurrence and intensity in which anxiety is performed throughout the course of the study, does reveal a body of students for whom the significant emotional indicator for how they perceive their learning, is negative. While a taxonomy of control may be deemed a strategy by which to regulate the cognisant emotional state of the student, nonetheless, the reflection placed on learning, is initiated from a place of negativity. This is in contrast to the easy smiles that are observed in the visual analysis of a significant number of the selfies posted. While happiness and enjoyment might be interpreted from these visual messages, enjoyment of the subject as an emotional indicator does not reveal itself in any of the textual descriptions. Where more positive annotations are reported, this is in relation to an 
anticipation that more positive emotions may be felt once the coursework has been completed or when the semester is over. What is not identified within any of the reflexive comments, is the link between academic performance and the enjoyment or interest in the subject. This may suggest that the students as a cohort, place less emotional value on whether or not the they are enjoying the subject; in this study the emotional engagement with learning is dominated by a desire to achieve academic success.

\section{Conclusions}

This study leverages the significance of the role images and photographs play in postmodern knowledge production, and using a methodology that promotes the idea of metadata as traces of human behaviour, reveals new opportunities that social networks and their technologies can provide in pedagogic research.

As a directed, closed-context and situated study of the selfie, the alternate performances of the selfie that have been uniquely revealed by the participants in this study as either oppositional or indexical, position the selfie as both an effective visual signifier upon which the self can become known, and an embodied communicative act in which the known self is constructed. The self awareness that is needed as the point of departure for the principle skills required to effectively perform the reflective process (critical analysis, synthesis and evaluation), is initiated by the selfie through two different strategies; by looking into the self image to understand what can become known about the self, and through the process of constructing the image to visualise what is already known about the self. In the former strategy, self awareness is brought into being by the selfie. Critical analysis is then performed, and what becomes known through this process becomes knowledge of the self synthesised through the annotations and metadata posted alongside the image. In the latter, the selfie as a material practice, is the outcome of the entirety of the reflective process. These alternate modes of self-reflection present new variables in understanding the selfie as a 
cultural artefact and highlight how the relationship between the selfie-taker and the selfimage is critical in examining the selfie as a reflexive sign.

The reflexive signs that have been under investigation in this study, have enabled issues and indicators that are important to the student to be revealed. Deploying the selfie as the visual evidence to prompt and communicate an understanding of the observed self, has provided a mechanism by which auto-ethnographic narratives of real-life learning experiences can be connected to theoretical strands of pedagogic practice. Within the framework of reflexivity created by the posting of the selfie, the study reveals the importance that students place on their emotional involvement with their learning, and presents new knowledge of the nuances created by applying gender as an analysis mode.

The study also reveals that each student through the course of their studies, experiences a variety and diversity of competing emotions: one particular performance across the semester communicated a range of messages including anxieties, aspirations, a sense of belonging, and withdrawal. This clearly indicates that any quality assurance metric applied at a specific moment within the academic calendar is unable to capture student behaviours that operate as something more multidimensional. In contrast the selfie-reflexive methodology can provide time-relevant data on student engagement that can subsequently be used diagnostically by instructors and course teams to identify individual student's immediate emotional response to their learning, and enable them to create transformational interventions that resolve, regulate and promote the dynamic process that is student engagement.

\section{Further research}

The institution at which this research was undertaken, Ulster University, has a reputation for widening access to higher education across Northern Ireland (Osborne 2006). As the institution has a record of sustained recruitment of NI-domiciled students from socially and economically disadvantaged backgrounds (McAlister, 2016), the objectified self 
observed in this study, provides an important and critical framework for understanding engagement with students that are under represented in the UK higher education sector. While this single narrow-angled snapshot study is able to identify the complexity of the reflexive construct, comparative reflexive studies with pre-1992 (higher tariff and relatively elite) institutions in the UK, and also those institutions in which race and ethnicity are more established than in the Northern Irish demographic, are needed to examine the impact of the socio-cultural context in which the auto-ethnographic narratives constructed through the selfie reflection, are being performed.

\section{Bibliography}

Algan, Ece. 2009. "What of Ethnography?” Television \& New Media 10(1): 7-9.

Astin, Alexander. W. 1999. "Student Involvement: A Developmental Theory for Higher Education.” Journal of College Student Development 40(5): 518-29.

Atkins, Sue and Kathy Murphy. 1993. "Reflection: A Review of the Literature." Journal of Advanced Nursing 18(8): 1188-1192 url: http://onlinelibrary.wiley.com/doi/10.1046/j.13652648.1993.18081188.x/epdf.

Barnard, Stephen R. 2016. "Spectacles of Self(ie) Empowerment? Networked Individualism and the Logic of the (Post)Feminist Selfie." In Communication and Information Technologies Annual, edited by Laura Robinson, Jeremy Schulz, Shelia R. Cotten, Timothy M. Hale, Apryl A. Williams and Joy L. Hightower, 63 - 88. Emerald Group Publishing Limited. Burns, Anne L. 2015. "Self(ie)-Discipline: Social Regulation as Enacted Through the Discussion of Photographic Practice.” International Journal of Communication. ISSN 19328036 (In Press) http://eprints.whiterose.ac.uk/83965/

Byrne, Marann and Barbara Flood. 2005. “A Study of Accounting Students' Motives, Expectations and Preparedness for Higher Education." Journal of Further and Higher Education 29 (2): 111-124 doi: 10.1080/03098770500103176.

Bryson, Colin, ed. 2014. Understanding and Developing Student Engagement. Abingdon: Routledge.

Chen, Hsin-Yu, Careen Yarnal, John T. P. Hustad and Damon Sims. 2016. "Take a Selfie of Life: A Qualitative Exploration of College Students' Self-Reflections on Free Time Use and 
Personal Values." Journal of College and Character 17(2): 101-115 doi:

10.1080/2194587X.2016.1159226.

Coates, Hamish. 2005. "The Value of Student Engagement for Higher Education Quality Assurance.” Quality in Higher Education 11(1): 25-36 doi:10.1080/13538320500074915.

Coleman, E. Gabriella. 2010. "Ethnographic Approaches to Digital Media.” Annual Review of Anthropology 39: 487-505. doi: 10.1146/annurev.anthro.012809.104945.

Cohen, Rachel, Toby Newton-John, and Amy Slater. 2018. "'Selfie'-Objectification: The Role of Selfies in Self-Objectification and Disordered Eating in Young Women." Computers in Human Behavior 79: 68-74 https://doi.org/10.1016/j.chb.2017.10.027

Cruz, Edgar Gómez and Helen Thornham. 2015. "Selfies Beyond Self- Representation: The (Theoretical) F(r)ictions of a Practice." Journal of Aesthetics \& Culture 7(1) 28073. doi: 10.3402/jac.v7.28073.

Cuddy, Amy. 2012. Your Body Language Shapes Who You Are. TEDGlobal (video). https://www.ted.com/talks/amy cuddy your body language shapes who you are.

Dewey, John. 1933. How We Think: A Restatement of the Relation of Reflective Thinking to the Educative Process. Lexington, Mass.: D.C. Heath and Company.

Dobson, Amy Shields. 2015. Postfeminist Digital Cultures: Femininity, Social Media, and Self-Representation. Palgrave Macmillian.

Duguay, Stefanie. 2016. “Lesbian, Gay, Bisexual, Trans, and Queer Visibility Through Selfies: Comparing Platform Mediators Across Ruby Rose's Instagram and Vine Presence." Social Media + Society 1-12 doi: 10.1177/2056305116641975.

Foucault, Michel. 1988. Technologies of the Self. In Technologies of the Self: A Seminar with Michel Foucault, edited by Luther H. Martin, Huck Gutman and Patrick H. Hutton, 16-49, London Tavistock.

Frosh, Paul. 2015. “The Gestural Image: The Selfie, Photography Theory, and Kinesthetic Sociability." International Journal of Communication 9: 1607-1628. url: http://ijoc.org/index.php/ijoc/article/viewFile/3146/1388.

Grushka, Kathryn. 2010. “Conceptualising Visual Learning as an Embodied and Performative Pedagogy for all Classrooms." Encounters in Theory and History of Education 11: 13-23. url:https://ojs.library.queensu.ca/index.php/encounters/article/view/3167.

Goffman, Erving. 1971. The Presentation of Self in Everyday Life. Harmondsworth: Penguin 
Hand, Martin. 2012. Ubiquitous Photography. Polity: Cambridge, UK and Malden, MA. Harford, Judith and Gerry MacRuairc. 2008. "Engaging Student Teachers in Meaningful Reflective Practice.” Teaching and Teacher Education 24:1884-1892.

Henley, Nancy M. 1977. Body Politics: Power, Sex and Nonverbal Communication. Englewood Cliffs, NJ: Prentice-Hall.

Ibrahim, Yasmin. 2015. "Self-Representation and the Disaster Event: Self-Imaging, Morality and Immortality." Journal of Media Practice 16(3): 211-227. doi:

10.1080/14682753.2015.1116755.

Jang, Jin Yea, Kyungsik Han, Patrick C. Shih and Dongwon Lee. 2015. "Generation Like: Comparative Characteristics in Instagram.” CHI '15 Proceedings of the 33rd Annual ACM Conference on Human Factors in Computing Systems. 4039-4042 url:

http://dx.doi.org/10.1145/2702123.2702555

Jerslev, Anne and Mette Mortensen. 2016. "What is the Self in the Celebrity Selfie?

Celebrification, Phatic Communication and Performativity." Celebrity Studies 7(2): 249-263. doi: 10.1080/19392397.2015.1095644.

Jones, Peter Reece. 1995. "Hindsight Bias in Reflective Practice: An Empirical Investigation." Journal of Advanced Nursing 21: 783-788. doi: 10.1046/j.13652648.1995.21040783.x.

Kahu, Ella R. 2013. "Framing Student Engagement in Higher Education.” Studies in Higher Education 38(5): 758-773. doi: 10.1080/03075079.2011.598505.

Kolisk, Michael and Jessica Roberts. 2015. "Selfies: Witnessing and Participatory Journalism with a Point of View." International Journal of Communication 9: 1672-1685.

Lister, Martin and Liz Wells. 2000. "Seeing Beyond Belief: Cultural Studies as an Approach to Analysing the Visual." In, The Handbook of Visual Analysis, edited by Theo Leeuwen and Carey Jewit, 61-91. London: Sage.

McAlister, Denise. 2016. Ulster University Widening Access and Participation Plan 2017-18. url: http://addl.ulster.ac.uk/wap

McIntosh, Paul. 2010. Action Research and Reflective Practice: Creative and Visual Methods to Facilitate Reflection and Learning. Abington; Routledge.

MacCann, Carolyn, Gerard J. Fogarty, Moshe Zeidner and Richard D. Roberts. 2011. 
"Coping Mediates the Relationship Between Emotional Intelligence (EI) and Academic Achievement." Contemporary Educational Psychology 36: 60-70. url:

http://isiarticles.com/bundles/Article/pre/pdf/73592.pdf.

Maniou, Theodora A. and Andreas Veglis. 2016. “'Selfie Journalism': Current Practices in Digital Media." Studies in Media and Communication 4(1) url: http://smc.redfame.com. Mascheroni, Giovanna, Jane Vincent and Estefania Jimenez. 2015. "Girls are Addicted to Likes so They Post Semi-Naked Selfies": Peer Mediation, Normativity and the Construction of Identity Online. Cyberpsychology: Journal of Psychosocial Research on Cyberspace 9(1) article 5. url: http://dx.doi.org/10.5817/CP2015-1-5.

Mirzoeff, Nicholas. 2016. How to See the World: An Introduction to Images, from SelfPortriats to Selfies, Maps to Movies and more. Basic Books.

Murray, Derek Conrad. 2015. "Notes to Self; The Visual Culture of Selfies in The Age of Social Media." Consumption Markets \& Culture 18(6): 490 - 516. doi:

10.1080/10253866.2015.1052967.

Osborne, Robert D. 2006. “Access to and Participation in Higher Education in Northern Ireland." Higher Education Quarterly 60(4): 333-348.

Paulhus, Delroy L. 1984. Two-Component Models of Socially Desirable Responding. Journal of Personality and Social Psychology 46: 598-609. url:

http://www2.psych.ubc.ca/ dpaulhus/research/SDR/downloads/ARTICLES/JPSP.1984.pdf. Pink, Sarah. 2001. Doing Visual Ethnography: Images, Media and Representation in Research. London: SAGE

Porter, Stephen R. 2011. "Do College Student Surveys Have Any Validity?” The Review of Higher Education 35(1): 45-76. doi: 10.1353/rhe.2011.0034.

Pounders, Kathrynn, Christine M. Kowalczyk and Kirsten Stowers. 2016. "Insight into the Motivation of Selfie Postings: Impression Management and Self-Esteem." European Journal of Marketing 50(9/10): 1879-1892. url: https://doi.org/10.1108/EJM-07-2015-0502.

Robbins, Kevin. 1996. Into the Image: Cultural Politics in the Field of Vision. Taylor \& Francis Group.

Rose, Gillian. 2016. Visual Methodologies: An Introduction to Researching with Visual Materials. Los Angeles; London: SAGE

Schleser, Max. 2014. "Connecting through Mobile Autobiographies: Self-Reflexive Mobile Filmmaking, Self-Representation, and Selfies.” In Mobile Media Making in an Age of 
Smartphones, edited by Marsha Berry and Max Schleser, 148-158. Palgrave Pivot, New York.

Schon, Donald A. 1987. Educating the Reflective Practitioner; Toward a New Design for Teaching and Learning in the Professions. San Francisco: Jossey-Bass Publishers.

Schutz, Paul A. and Heather A. Davis. 2000. "Emotions and Self-Regulation During Testtaking.” Educational Psychologist 35: 243-256. doi:10.1207/S15326985EP3504_03.

Skinner, Ellen A. 1996. “A Guide to Constructs of Control.” Journal of Personality and Social Psychology 71: 549-570. url:

https://www.pdx.edu/sites/www.pdx.edu.psy/files/A\%20guide $\% 20$ to $\% 20$ constructs $\% 20$ of $\% 2$ 0control--Skinner--1996.pdf.

OkLyu, Seong. 2016. “Travel Selfies on Social Media as Objectified Self-Presentation.” Tourism Management 54: 185-195. url: https://doi.org/10.1016/j.tourman.2015.11.001. Sorokowska, Agnieszka, Anna Oleszkiewicz, Tomasz Frackowiak, Katarzyn Pisanski, Anna Chmiel and Piotr Sorokowski. 2016. "Selfies and Personality: Who Posts Self-Portrait Photographs?" Personality and Individual Differences 90:119-123. url:

https://doi.org/10.1016/j.paid.2015.10.037

Sorokowski, Piotr, Agnieszka Sorokowska, Anna Oleszkiewicz and Katarzyna Pisanski. 2015. "Selfie Posting Behaviors are Associated with Narcissism Among Men." Personality and Individual Differences 85:123-127. doi: 10.1016/j.paid.2015.05.004.

Spence, Jo and Patricia Holland, eds. 1991. Family Snaps: The Meaning of Domestic Photography. London: Virago.

Sung, Yongjun, Jung-Ah Lee, Eunice Kim and Sejung Marin Choi. 2016. "Why We Post Selfies: Understanding Motivations for Posting Pictures of Oneself." Personality and Individual Differences 97: 260-265. url: https://doi.org/10.1016/j.paid.2016.03.032.

Toffoletti, Kim and Holly Thorpe. 2018. "Female Athletes' Self-representation on Social Media: A Feminist Analysis of Neoliberal Marketing Strategies in 'Economies of Visibility." Feminism \& Psychology 28(1): 11-31.

Trowler, Vicki. 2010. Student Engagement Literature Review. York: Higher Education Academy. url:

https://www.heacademy.ac.uk/system/files/studentengagementliteraturereview_1.pdf Van Dijck, Jose. 2013. The Culture of Connectivity: A Critical History of Social Media. Oxford University Press. 
Van Manen, Max. 1995. "On the Epistemology of Reflective Practice.” Teachers and Teaching: Theory and Practice 1(1): 33-50. doi: 10.1080/1354060950010104.

Wargo, Jon M. 2017. “Every Selfie Tells a Story ...:LGBTQ Youth Lifestreams and New Media Narratives as Connective Identity Texts." New Media \& Society 19(4): 560- 578. url: https://doi.org/10.1177/1461444815612447.

Williams, Apryl A. and Beatriz Aldana Marquez. 2015. "The Lonely Selfie King: Selfies and the Conspicuous Prosumption of Gender and Race." International Journal of Communication 9: $1775-1787$.

Williams, Zoe. 2016. "Me! Me! Me! Are we living through a narcissism epidemic?” The Guardian, March 2. url:

https://www.theguardian.com/lifeandstyle/2016/mar/02/narcissism-epidemic-self-obsessionattention-seeking-oversharing

Zappavigna, Michele. 2016. "Social Media Photography: Construing Subjectivity in Instagram Images.” Visual Communication 15(3): 271-292.

Zepke, Nick and Linda Leach. 2010. "Improving Student Engagement: Ten proposals for Action." Active Learning in Higher Education 11(3): 167-177. doi:

$10.1177 / 1469787410379680$.

Zhao, Chun-Mei and George D. Kuh. 2004. "Adding Value: Learning Communities and Student Engagement." Research in Higher Education 45(2): 115-38. url:

http://dx.doi.org/10.1023/B:RIHE.0000015692.88534.de.

\section{Table 1: Practice of the Selfie, from object to subject}

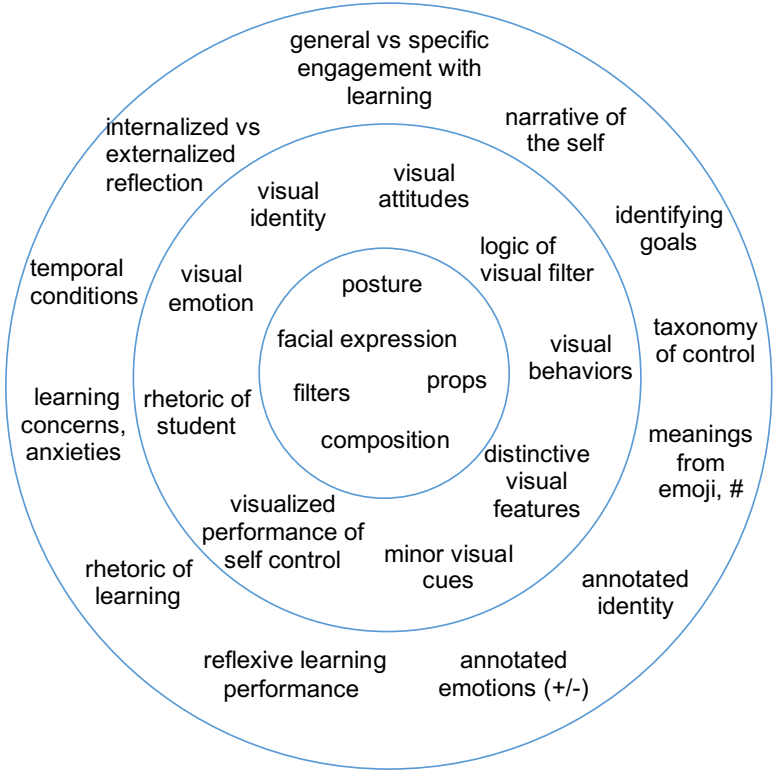


Table 2: Selfie Reflection and Learning Engagement Matrix

\begin{tabular}{|c|c|c|c|c|c|c|c|c|c|c|c|c|}
\hline $\begin{array}{c}\text { Selfie } \\
\text { Reflection }\end{array}$ & 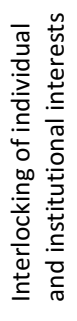 & 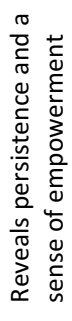 & 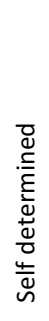 & 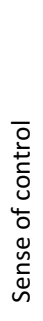 & 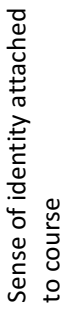 & 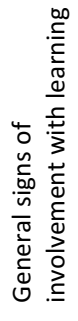 & 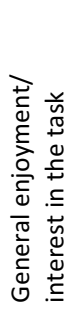 & 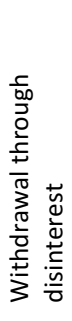 & 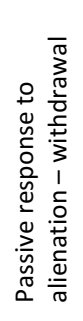 & 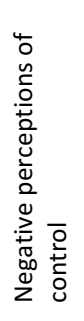 & 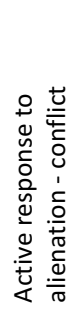 & 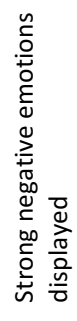 \\
\hline Hair pulling time & & & & & & & & & & & & \\
\hline $\begin{array}{l}\text { Feeling positive } \\
\text { this week after } \\
\text { coming up with } \\
\text { project ideas }\end{array}$ & & & & & & & & & & & & \\
\hline
\end{tabular}

Picture 1 Oppositional Selfie Practice

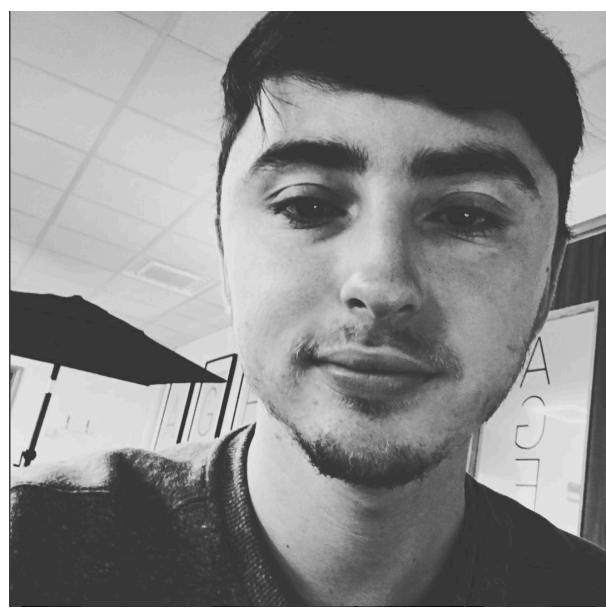

Picture 2 Indexical Selfie Practice

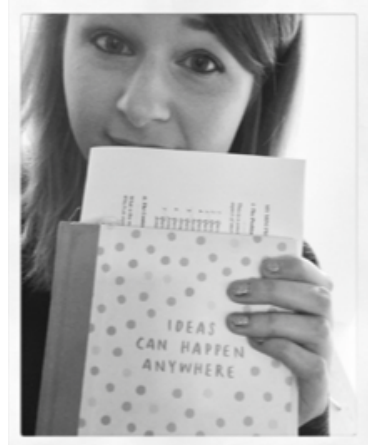


Picture 3 Starting to feel a little more under pressure

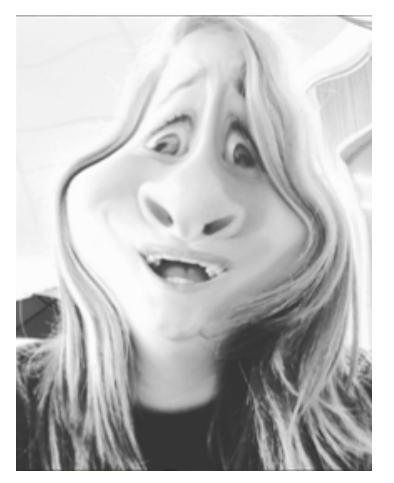

Picture 4 Female Selfie

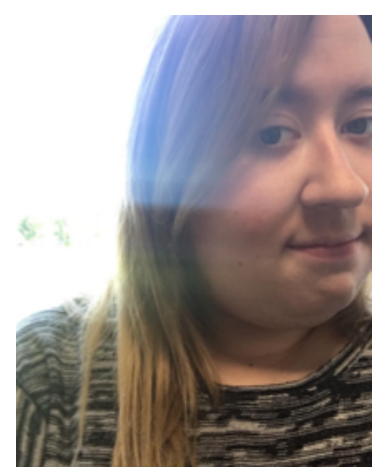

Picture 5 Male Selfie

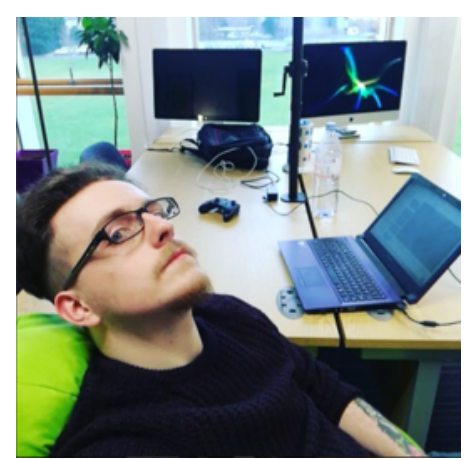

Picture 6 Shared Selfie Performances 